\title{
A Metallacage Encapsulating Chloride as a Probe for a Solvation Scale in Ionic Liquids
}

Corinne Daguenet and Paul J. Dyson

Institut des Sciences et Ingénierie Chimiques, Ecole Polytechnique Fédérale de Lausanne (EPFL), CH-1015 Lausanne, Switzerland. E-mail: paul.dyson@epfl.ch 


\section{Supporting information}

Details for the derivation of equation (1)-(5)

For a solution of $\mathbf{1}$ in which the analytical concentration of $\mathbf{1}$ equals $c$, the equilibrium constant for the dissociation of the cage $\mathbf{1}$ into its components is expressed as follows, where $c-\chi=[c]$ represents the concentration of $\mathbf{1}$ at equilibrium.

$K=\frac{(4 x)^{4} \cdot(2 x)^{2} \cdot x}{c-x}=\frac{1024 x^{7}}{c-x}=\frac{1024(c-[c])^{7}}{[c]}$

As $[c]$ can be expressed as a function of the absorbance according to Lambert-Beer's Law (optical pathlength equals $1 \mathrm{~cm}$ ), it follows:

$K=\frac{1024\left(c-\frac{A}{\varepsilon}\right)^{7}}{\frac{A}{\varepsilon}}$

$\ln K=\ln 1024+7 \cdot \ln \left(c-\frac{A}{\varepsilon}\right)-\ln \left(\frac{A}{\varepsilon}\right)=7 \cdot \ln (c \cdot \varepsilon-A)-\ln A-6 \cdot \ln \varepsilon+\ln 1024$

Using Van’t Hoff equation, it follows:

$\frac{1}{T}=-\frac{R}{\Delta H}\left(7 \cdot \ln (c \cdot \varepsilon-A)-\ln A-6 \cdot \ln \varepsilon+\ln 1024-\frac{\Delta S}{R}\right)$

As for each ionic liquid two dilutions were prepared from a stock solution of known concentration, $c_{0}$, the analytical concentration, $c$, equals $c_{0} \times d_{i}$, where $d_{i}$ is the dilution factor for the dilution i. Sets of temperatures and absorbances corresponding to a dilution i are then expressed as follows:

$\frac{1}{T_{i}}=-\frac{R}{\Delta H}\left(7 \cdot \ln \left(c_{0} \cdot d_{i} \cdot \varepsilon-A_{i}\right)-\ln A_{i}-6 \cdot \ln \varepsilon+\ln 1024-\frac{\Delta S_{i}}{R}\right)$ 


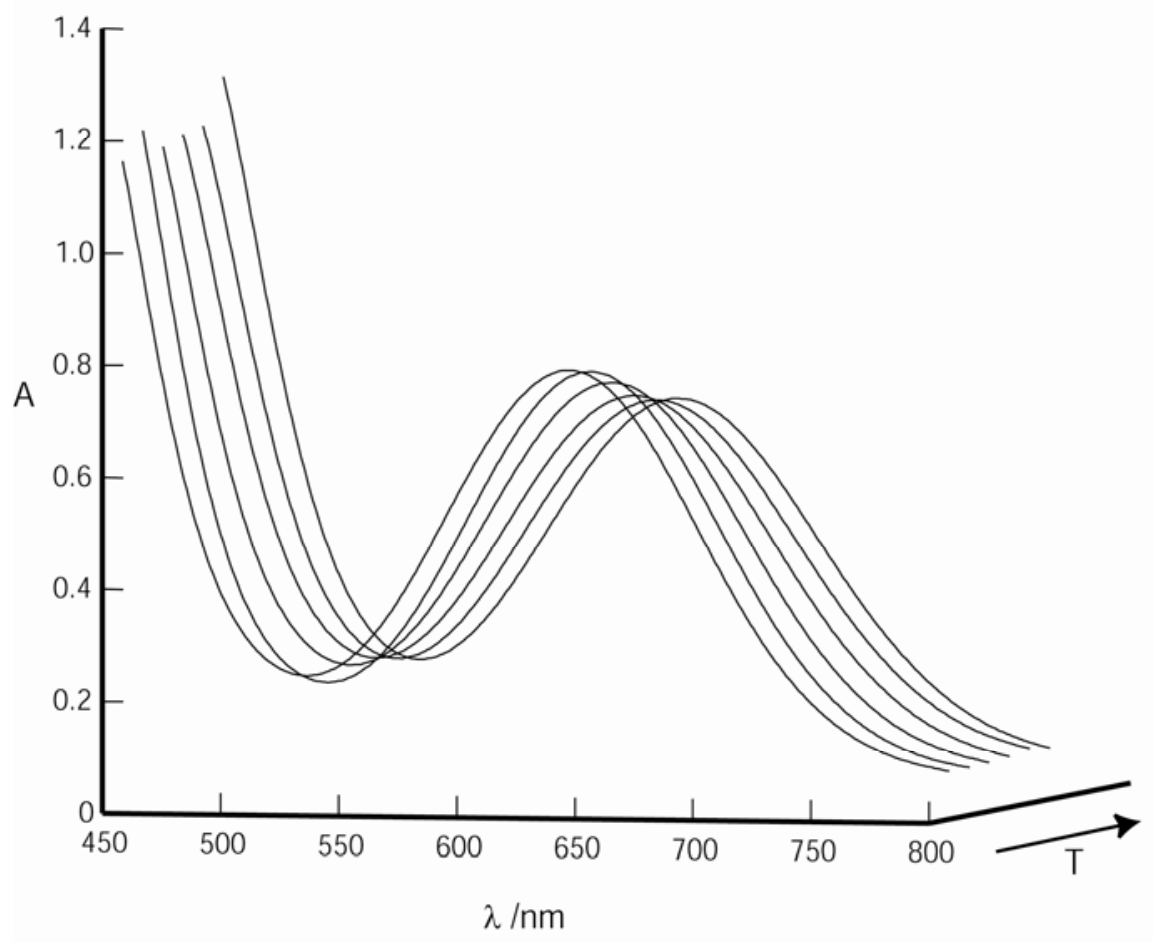

Figure S1. UV-visible spectra of $\mathbf{1}$ in $[\mathrm{bmim}]\left[\mathrm{Tf}_{2} \mathrm{~N}\right]$ at variable temperature. $\mathrm{c}(\mathbf{1})=0.45 \mathrm{mM}, \mathrm{T}=$ 18.7, 28.4, 38.3, 57.8, 67.6 and $77.3^{\circ} \mathrm{C}$. 


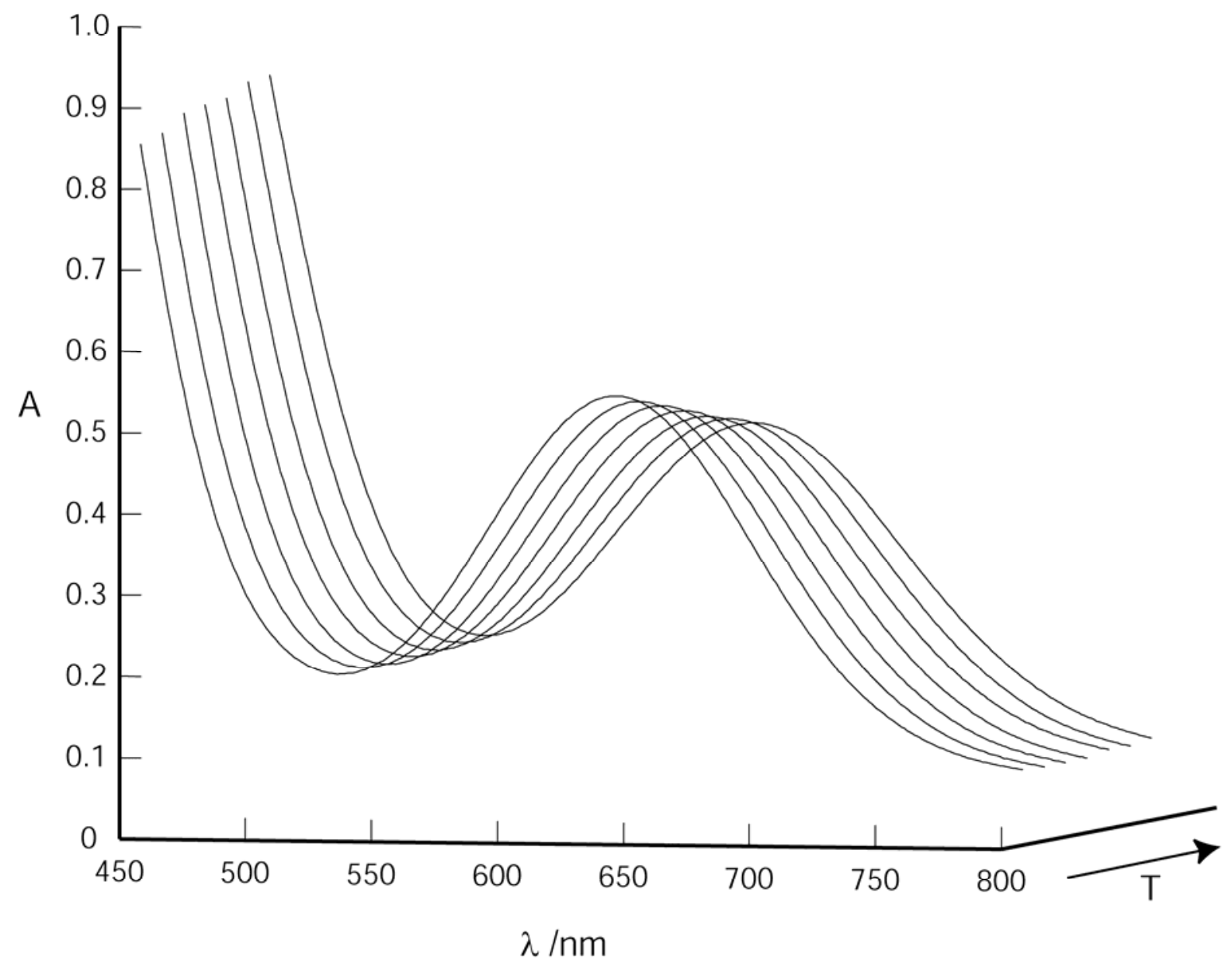

Figure S2. UV-visible spectra of $\mathbf{1}$ in $[\mathrm{bmim}]\left[\mathrm{Tf}_{2} \mathrm{~N}\right]$ at variable temperature. $\mathrm{c}(\mathbf{1})=0.29 \mathrm{mM}, \mathrm{T}=$ 18.7, 28.4, 38.3, 57.6, 67.1 and $77.1^{\circ} \mathrm{C}$. 


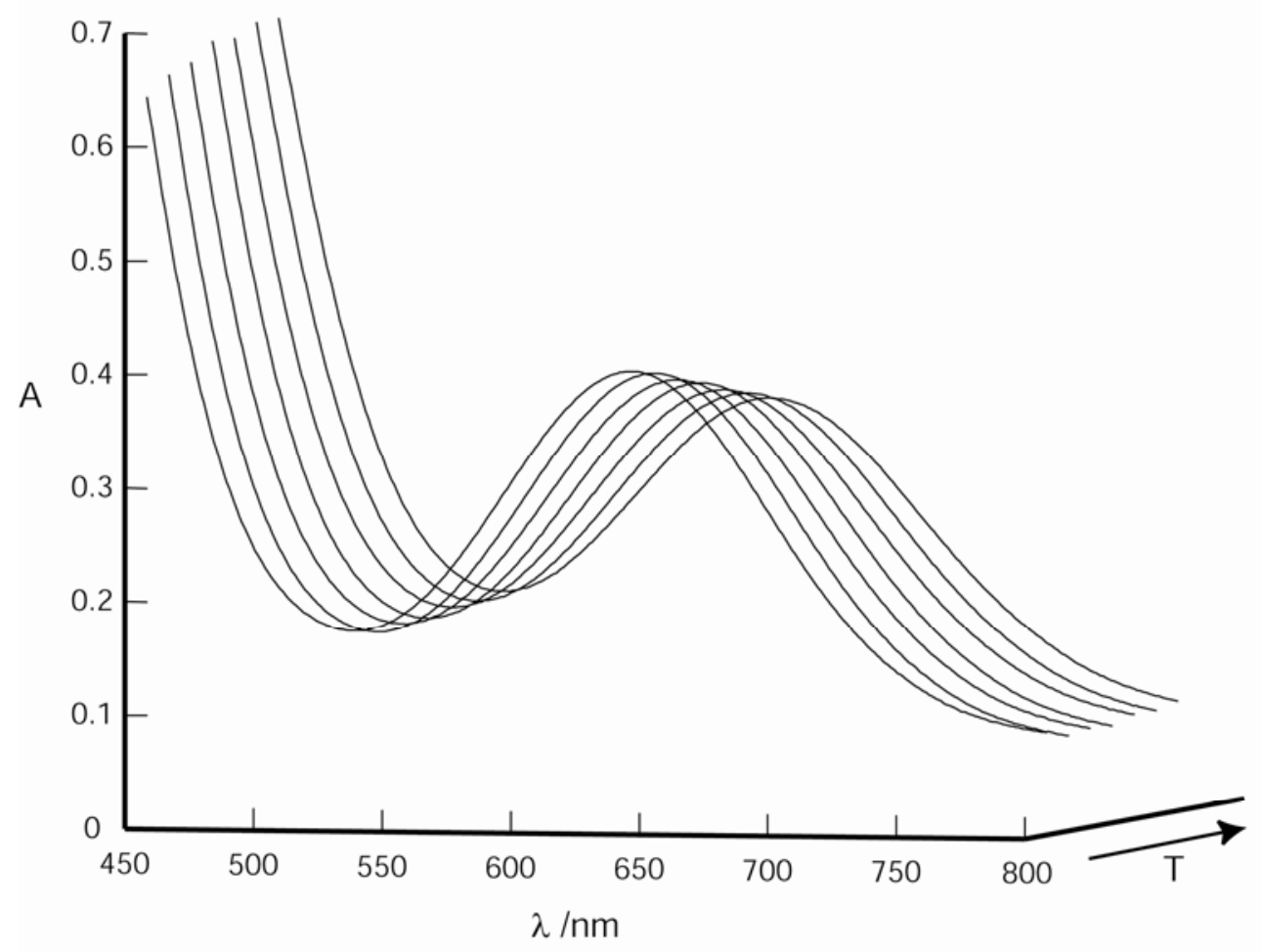

Figure S3. UV-visible spectra of $\mathbf{1}$ in $[\mathrm{bmim}]\left[\mathrm{Tf}_{2} \mathrm{~N}\right]$ at variable temperature. $\mathrm{c}(\mathbf{1})=0.21 \mathrm{mM}, \mathrm{T}=$ 18.3, 28.5, 38.2, 57.6, 67.4 and $77.1^{\circ} \mathrm{C}$.

Table S1 Absorbances at $638 \mathrm{~nm}$ of $\mathbf{1}$ dissolved in $[$ bmim $]\left[\mathrm{Tf}_{2} \mathrm{~N}\right]$.

\begin{tabular}{|rrrr|}
\hline $\mathrm{c}(\mathbf{1}) / \mathrm{mM}$ & $\mathrm{A}(638)$ & \multicolumn{1}{c|}{$1 / \mathrm{T}^{-} \mathrm{K}^{-1}$} & \multicolumn{1}{c|}{$\mathrm{T}{ }^{\circ} \mathrm{C}$} \\
\hline 0.45 & 0.7925 & 0.003426 & 18.7 \\
0.45 & 0.7561 & 0.003211 & 38.3 \\
0.45 & 0.7255 & 0.003022 & 57.8 \\
0.45 & 0.7100 & 0.002935 & 67.6 \\
0.45 & 0.7830 & 0.003316 & 28.4 \\
0.45 & 0.7052 & 0.002853 & 77.3 \\
\hline 0.29 & 0.5346 & 0.003316 & 28.4 \\
0.29 & 0.5126 & 0.003114 & 48.0 \\
0.29 & 0.4924 & 0.002939 & 67.1 \\
0.29 & 0.4820 & 0.002855 & 77.1 \\
0.29 & 0.5005 & 0.003023 & 57.6 \\
0.29 & 0.5239 & 0.003211 & 38.3 \\
0.29 & 0.5466 & 0.003426 & 18.7 \\
\hline 0.21 & 0.3985 & 0.003315 & 28.5 \\
0.21 & 0.3828 & 0.003113 & 48.1 \\
0.21 & 0.3891 & 0.003212 & 38.2 \\
0.21 & 0.3666 & 0.002936 & 67.4 \\
0.21 & 0.3589 & 0.002855 & 77.1 \\
0.21 & 0.3732 & 0.003023 & 57.6 \\
0.21 & 0.4035 & 0.003431 & 18.3 \\
\hline & & &
\end{tabular}




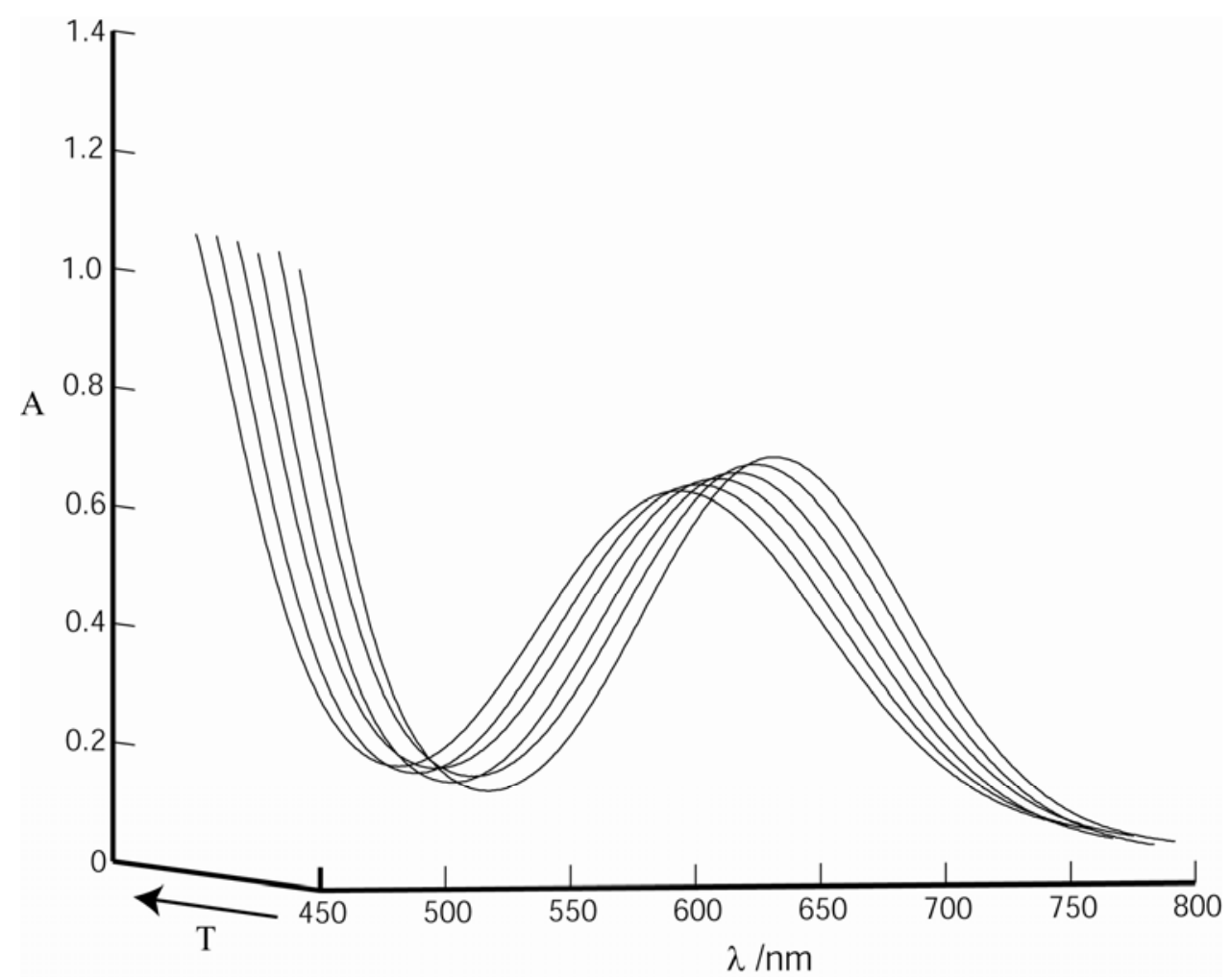

Figure S4. UV-visible spectra of $\mathbf{1}$ in $[\mathrm{bdmim}]\left[\mathrm{Tf}_{2} \mathrm{~N}\right]$ at variable temperature. $\mathrm{c}(\mathbf{1})=0.47 \mathrm{mM}, \mathrm{T}=$ 18.7, 28.4, 38.1, 48.0, 57.6 and $67.4{ }^{\circ} \mathrm{C}$.

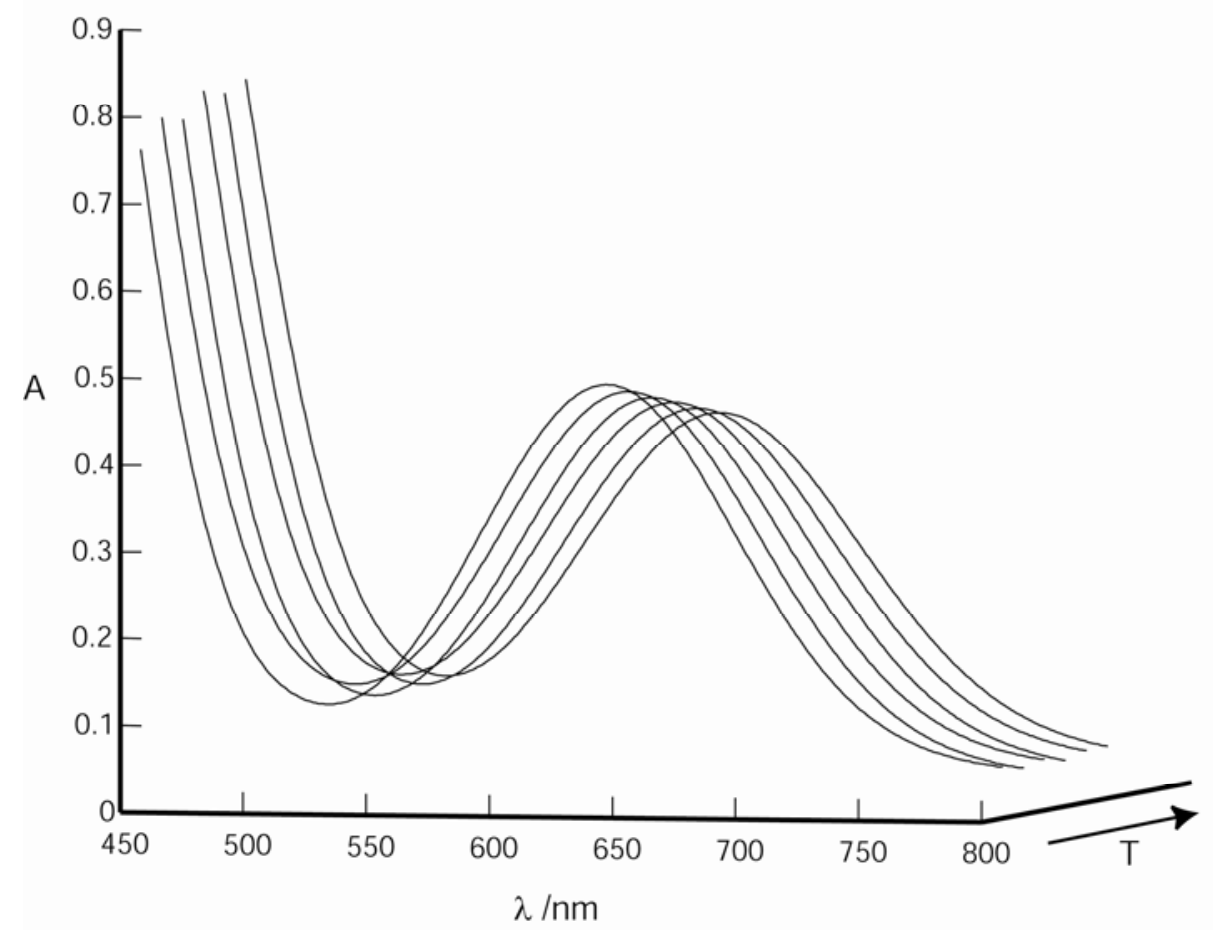

Figure S5. UV-visible spectra of $\mathbf{1}$ in $\left[\right.$ bdmim] $\left[\mathrm{Tf}_{2} \mathrm{~N}\right]$ at variable temperature. $\mathrm{c}(\mathbf{1})=0.30 \mathrm{mM}, \mathrm{T}=$ 18.7, 28.8, 38.1, 48.0, 57.5 and $67.4{ }^{\circ} \mathrm{C}$. 


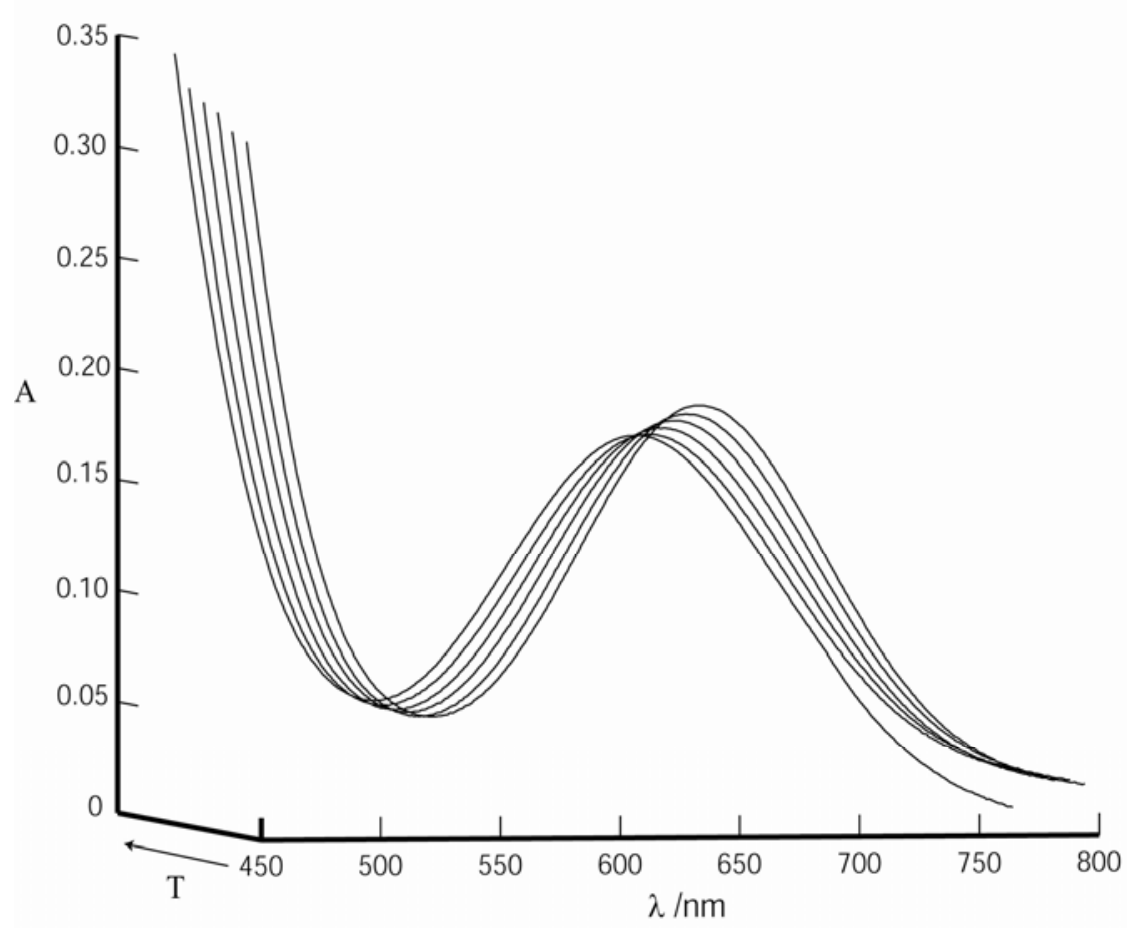

Figure S6. UV-visible spectra of $\mathbf{1}$ in $[\mathrm{bdmim}]\left[\mathrm{Tf}_{2} \mathrm{~N}\right]$ at variable temperature. $\mathrm{c}(\mathbf{1})=0.12 \mathrm{mM}, \mathrm{T}=$ 18.7, 28.3, 38.2, $47.9,57.7$ and $67.3^{\circ} \mathrm{C}$.

Table S2 Absorbances at $638 \mathrm{~nm}$ of $\mathbf{1}$ dissolved in [bdmim] $\left[\mathrm{Tf}_{2} \mathrm{~N}\right]$.

\begin{tabular}{|rrrr|}
\hline $\mathrm{C}(\mathbf{1}) / \mathrm{mM}$ & $\mathrm{A}(638)$ & $1 / \mathrm{T} / \mathrm{K}^{-1}$ & \multicolumn{1}{c|}{$/{ }^{\circ} \mathrm{C}$} \\
\hline 0.47 & 0.6997 & 0.003316 & 28.4 \\
0.47 & 0.6654 & 0.003114 & 48.0 \\
0.47 & 0.6342 & 0.002936 & 67.4 \\
0.47 & 0.6502 & 0.003023 & 57.6 \\
0.47 & 0.6809 & 0.003213 & 38.1 \\
0.47 & 0.7171 & 0.003426 & 18.7 \\
\hline 0.30 & 0.4812 & 0.003312 & 28.8 \\
0.30 & 0.4593 & 0.003114 & 48.0 \\
0.30 & 0.4377 & 0.002936 & 67.4 \\
0.30 & 0.4476 & 0.003024 & 57.5 \\
0.30 & 0.4694 & 0.003213 & 38.1 \\
0.30 & 0.4938 & 0.003426 & 18.7 \\
\hline 0.12 & 0.1927 & 0.003426 & 18.7 \\
0.12 & 0.1833 & 0.003212 & 38.2 \\
0.12 & 0.1745 & 0.003023 & 57.7 \\
0.12 & 0.1787 & 0.003115 & 47.9 \\
0.12 & 0.1876 & 0.003317 & 28.3 \\
0.12 & 0.1727 & 0.002937 & 67.3 \\
\hline
\end{tabular}




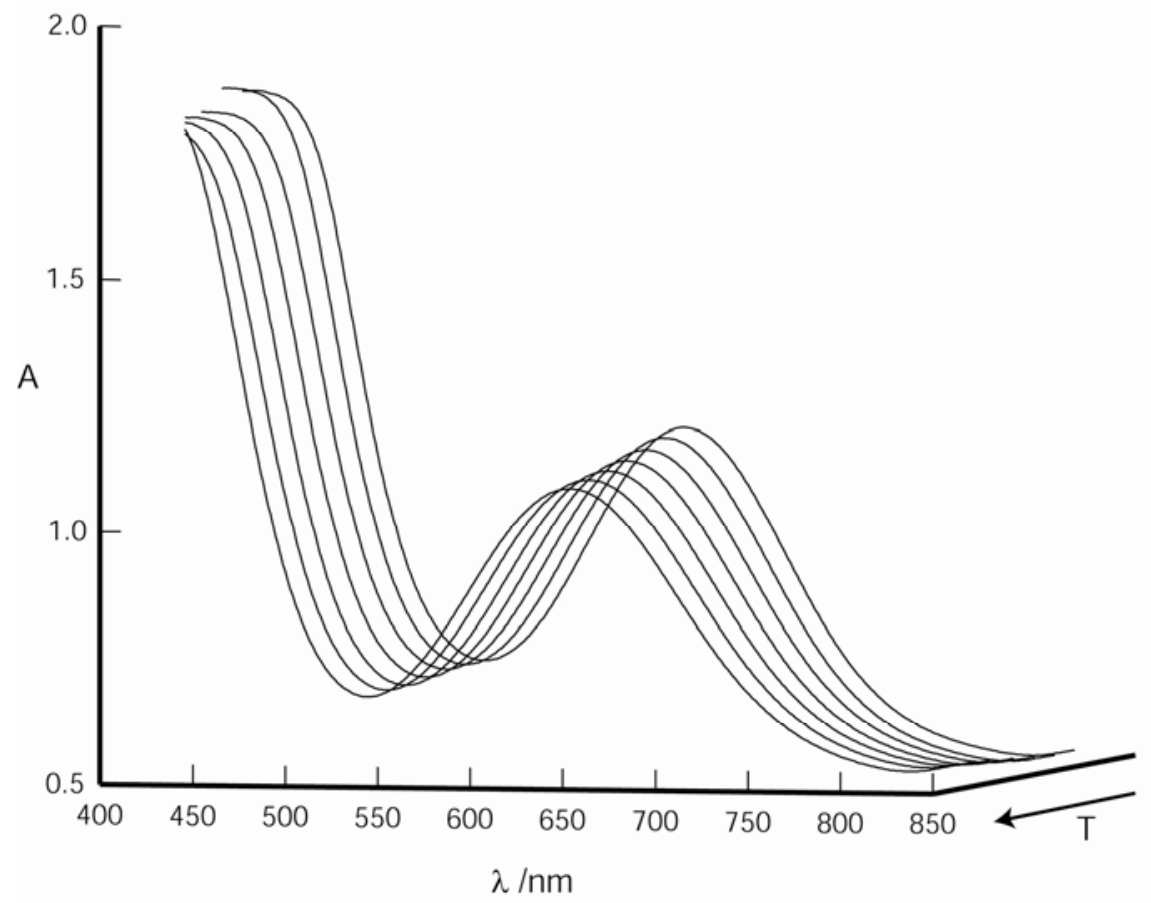

Figure S7. UV-visible spectra of $\mathbf{1}$ in $[\mathrm{bpy}]\left[\mathrm{Tf}_{2} \mathrm{~N}\right]$ at variable temperature. $\mathrm{c}(\mathbf{1})=0.39 \mathrm{mM}, \mathrm{T}=18.7$, 28.1, 37.1, 46.3, 55.2, 64.3 and $74.9^{\circ} \mathrm{C}$.

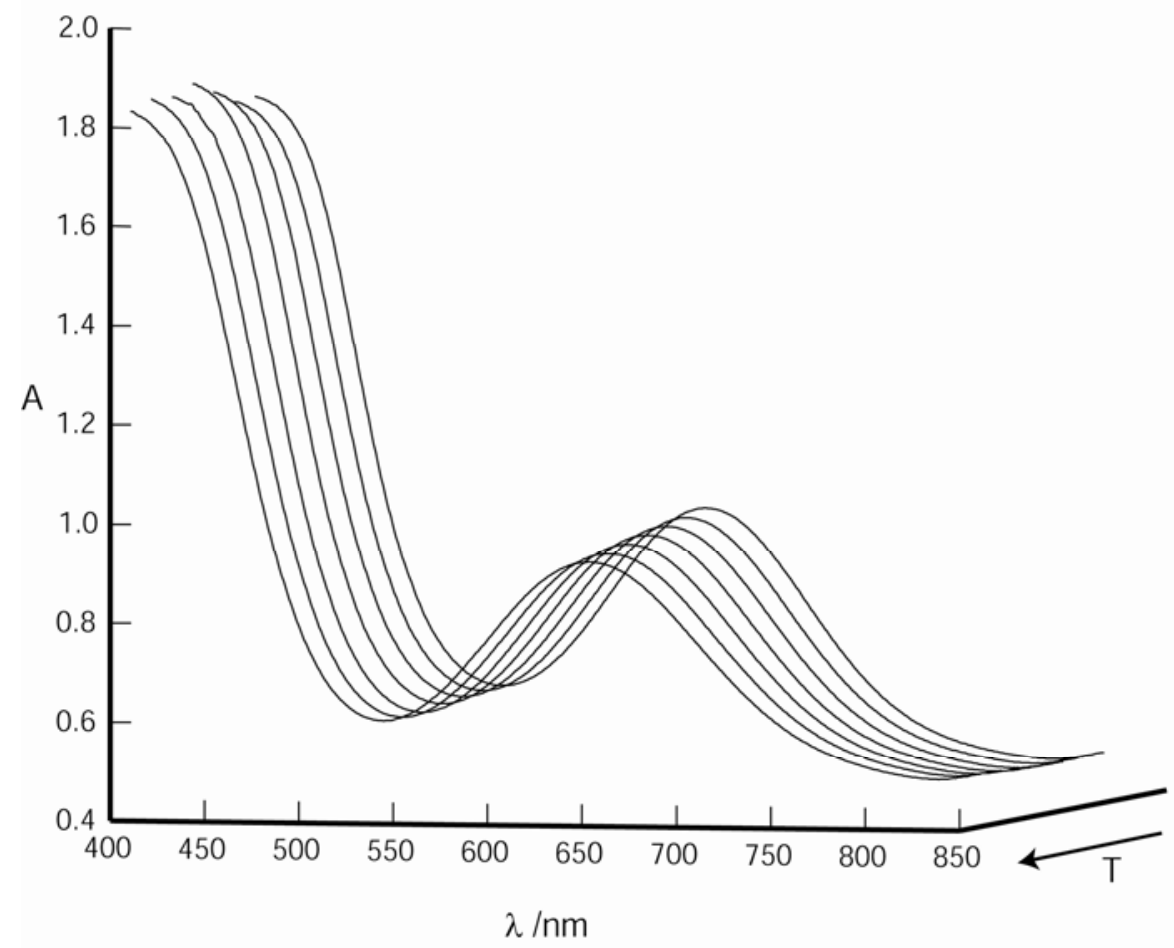

Figure S8. UV-visible spectra of $\mathbf{1}$ in $[\mathrm{bpy}]\left[\mathrm{Tf}_{2} \mathrm{~N}\right]$ at variable temperature. $\mathrm{c}(\mathbf{1})=0.29 \mathrm{mM}, \mathrm{T}=18.8$, 28.4, 37.5, 46.9, 56.1, 65.4 and $74.6^{\circ} \mathrm{C}$. 


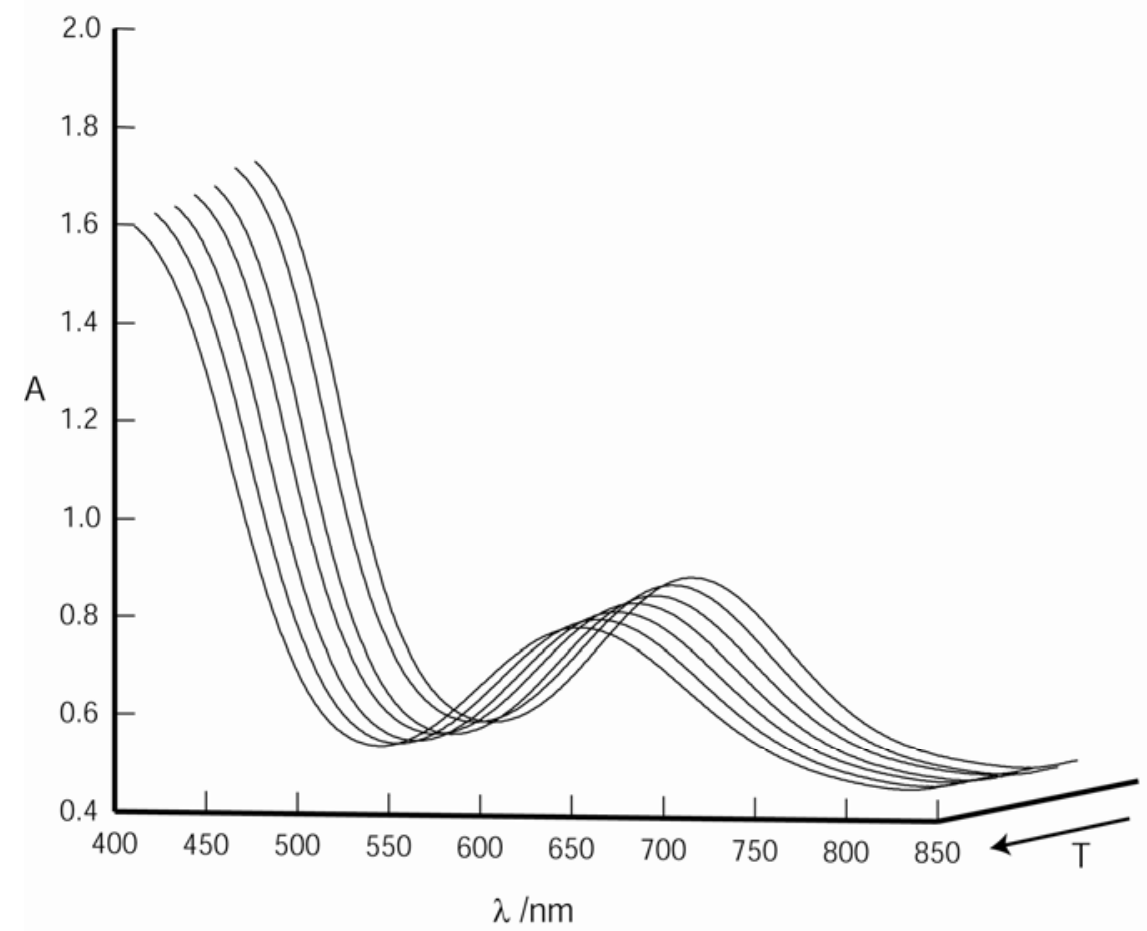

Figure S9. UV-visible spectra of $\mathbf{1}$ in $[\mathrm{bpy}]\left[\mathrm{Tf}_{2} \mathrm{~N}\right]$ at variable temperature. $\mathrm{c}(\mathbf{1})=0.24 \mathrm{mM}, \mathrm{T}=18.7$, 28.4, 38.1, 48.1, 57.8, 67.7 and $77.4^{\circ} \mathrm{C}$.

Table S3 Absorbances at $638 \mathrm{~nm}$ of $\mathbf{1}$ dissolved in [bpy][Tf $\left.{ }_{2} \mathrm{~N}\right]$.

\begin{tabular}{|rrrr|}
\hline $\mathrm{c}(\mathbf{1}) / \mathrm{mM}$ & $\mathrm{A}(638)$ & \multicolumn{1}{c|}{$1 / \mathrm{T} / \mathrm{K}^{-1}$} & $\mathrm{~T} /{ }^{\circ} \mathrm{C}$ \\
\hline 0.39 & 1.0856 & 0.002873 & 74.9 \\
0.39 & 1.0957 & 0.002963 & 64.3 \\
0.39 & 1.1062 & 0.003046 & 55.2 \\
0.39 & 1.1192 & 0.003130 & 46.3 \\
0.39 & 1.1334 & 0.003223 & 37.1 \\
0.39 & 1.1498 & 0.003320 & 28.1 \\
0.39 & 1.1623 & 0.003426 & 18.7 \\
\hline 0.29 & 0.9237 & 0.002876 & 74.6 \\
0.29 & 0.9330 & 0.002954 & 65.4 \\
0.29 & 0.9438 & 0.003037 & 56.1 \\
0.29 & 0.9549 & 0.003125 & 46.9 \\
0.29 & 0.9646 & 0.003219 & 37.5 \\
0.29 & 0.9744 & 0.003316 & 28.4 \\
0.29 & 0.9857 & 0.003425 & 18.8 \\
\hline 0.24 & 0.8234 & 0.003316 & 28.4 \\
0.24 & 0.8021 & 0.003113 & 48.1 \\
0.24 & 0.7845 & 0.002934 & 67.7 \\
0.24 & 0.7761 & 0.002853 & 77.4 \\
0.24 & 0.8311 & 0.003426 & 18.7 \\
0.24 & 0.8097 & 0.003213 & 38.1 \\
0.24 & 0.7921 & 0.003022 & 57.8 \\
\hline
\end{tabular}




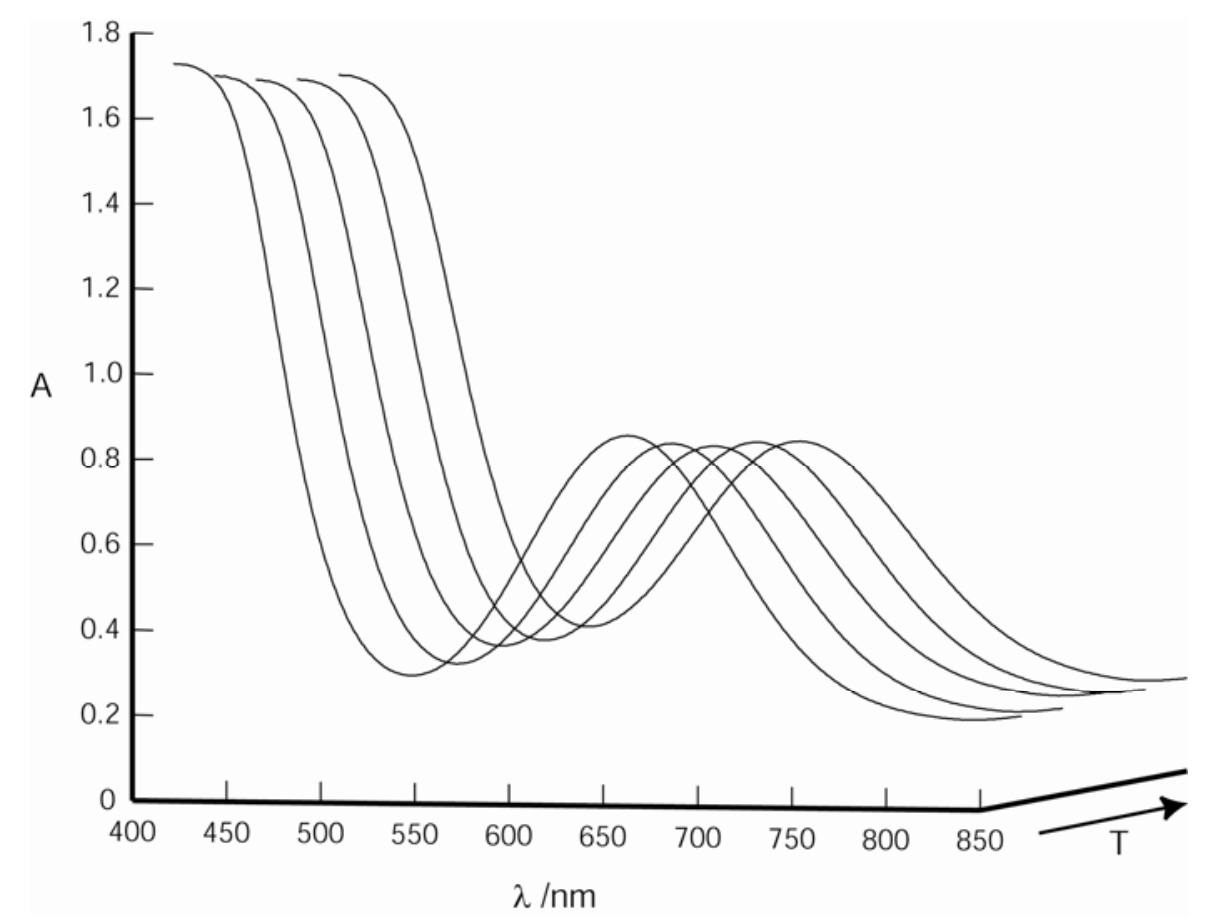

Figure S10. UV-visible spectra of $\mathbf{1}$ in $[$ bmpy $]\left[\mathrm{Tf}_{2} \mathrm{~N}\right]$ at variable temperature. $\mathrm{c}(\mathbf{1})=0.58 \mathrm{mM}, \mathrm{T}=$ 28.4, 48.0, 57.7, 67.6 and $77.3^{\circ} \mathrm{C}$.

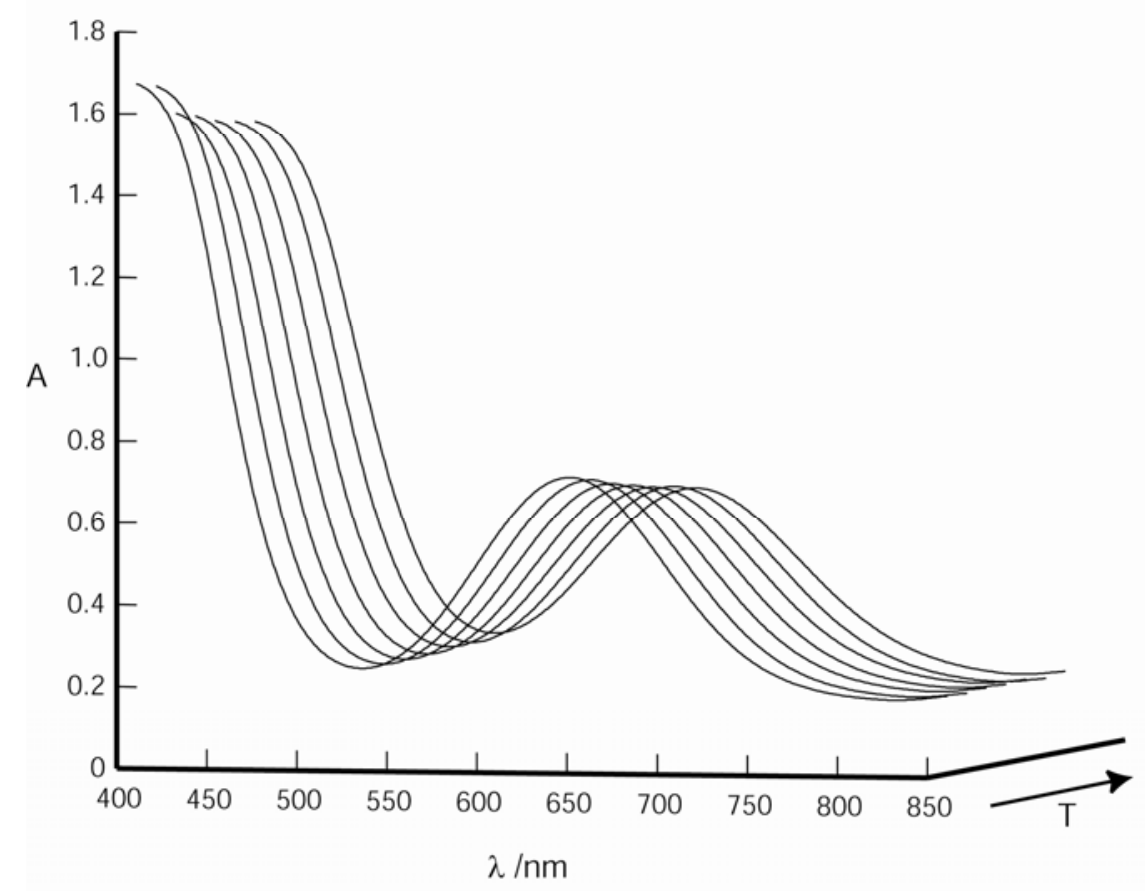

Figure S11. UV-visible spectra of $\mathbf{1}$ in $[\mathrm{bmpy}]\left[\mathrm{Tf}_{2} \mathrm{~N}\right]$ at variable temperature. $\mathrm{c}(\mathbf{1})=0.46 \mathrm{mM}, \mathrm{T}=$ 18.7, 28.6, 38.1, 47.9, 58.7, 67.5 and $77.2^{\circ} \mathrm{C}$. 


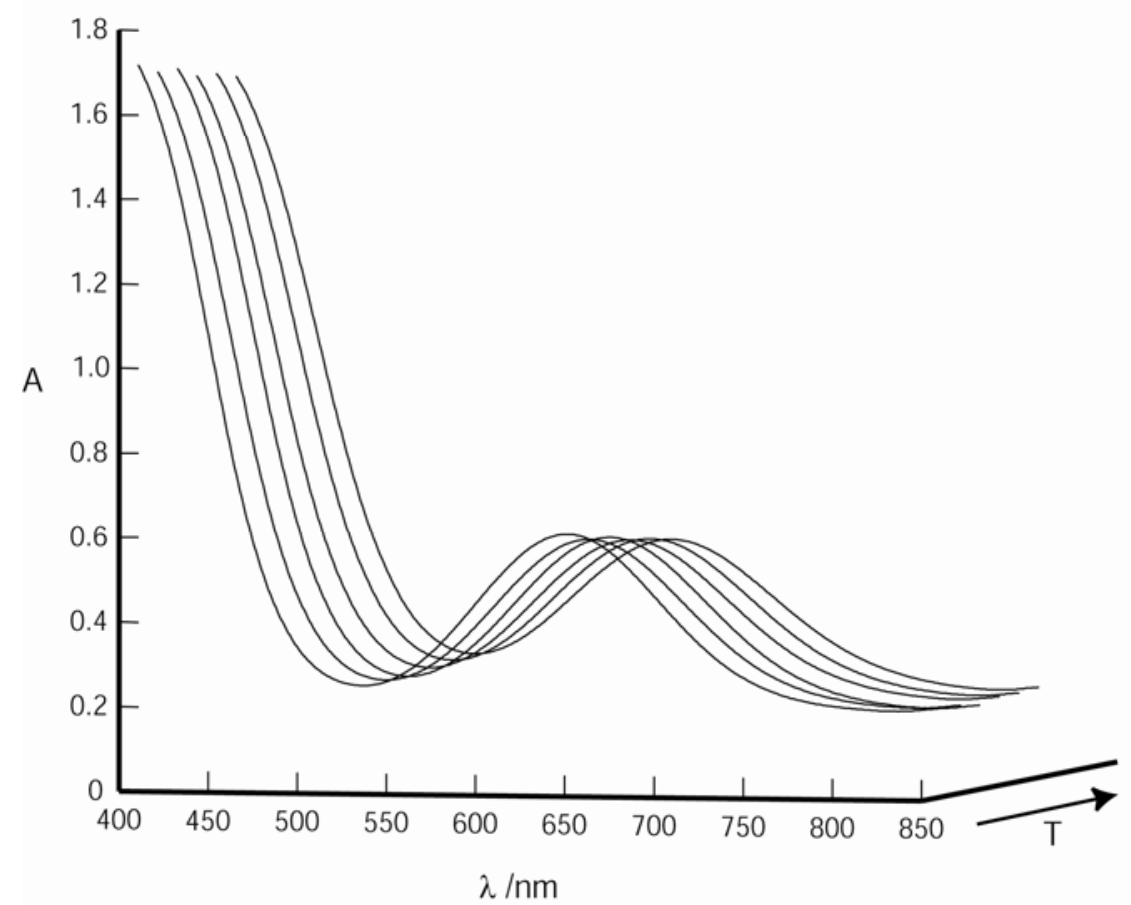

Figure S12. UV-visible spectra of $\mathbf{1}$ in $\left[\right.$ bmpy] $\left[\mathrm{Tf}_{2} \mathrm{~N}\right]$ at variable temperature. $\mathrm{c}(\mathbf{1})=0.35 \mathrm{mM}, \mathrm{T}=$ 18.7, 38.1, 48.0, 57.7, 67.5 and $77.2^{\circ} \mathrm{C}$.

Table S4 Absorbances at $638 \mathrm{~nm}$ of $\mathbf{1}$ dissolved in [bmpy][Tf $2 \mathrm{~N}]$.

\begin{tabular}{|rrrr|}
\hline $\mathrm{C}(\mathbf{1}) / \mathrm{mM}$ & $\mathrm{A}(638)$ & $1 / \mathrm{T} / \mathrm{K}^{-1}$ & $\mathrm{~T} /{ }^{\circ} \mathrm{C}$ \\
\hline 0.58 & 0.7749 & 0.002935 & 67.6 \\
0.58 & 0.7583 & 0.002853 & 77.3 \\
0.58 & 0.7847 & 0.003023 & 57.7 \\
0.58 & 0.8473 & 0.003316 & 28.4 \\
0.58 & 0.8093 & 0.003114 & 48.0 \\
\hline 0.46 & 0.6974 & 0.003314 & 28.6 \\
0.46 & 0.6651 & 0.003115 & 47.9 \\
0.46 & 0.6433 & 0.002936 & 67.5 \\
0.46 & 0.6301 & 0.002854 & 77.2 \\
0.46 & 0.6516 & 0.003013 & 58.7 \\
0.46 & 0.6786 & 0.003213 & 38.1 \\
0.46 & 0.7127 & 0.003426 & 18.7 \\
\hline 0.35 & 0.5607 & 0.002936 & 67.5 \\
0.35 & 0.5500 & 0.002854 & 77.2 \\
0.35 & 0.5669 & 0.003023 & 57.7 \\
0.35 & 0.5871 & 0.003213 & 38.1 \\
0.35 & 0.6091 & 0.003426 & 18.7 \\
0.35 & 0.5826 & 0.003114 & 48.0 \\
\hline
\end{tabular}




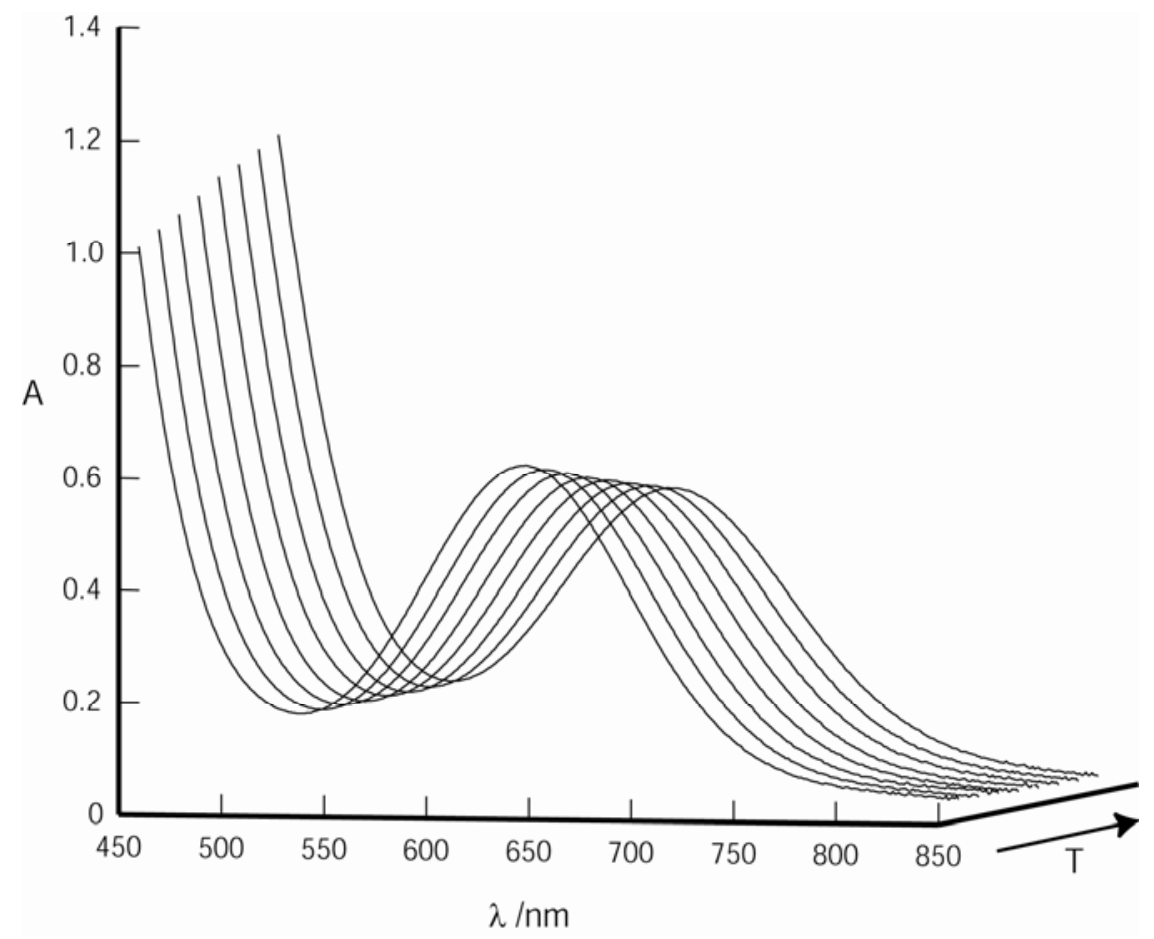

Figure S13. UV-visible spectra of $\mathbf{1}$ in $\left[\mathrm{C}_{5} \mathrm{e}_{3} \mathrm{am}\right]\left[\mathrm{Tf}_{2} \mathrm{~N}\right]$ at variable temperature. $\mathrm{c}(\mathbf{1})=0.37 \mathrm{mM}, \mathrm{T}=$ 9.8, 19.3, 28.8, 38.6, 48.1, 57.7, 67.2 and $76.8^{\circ} \mathrm{C}$.

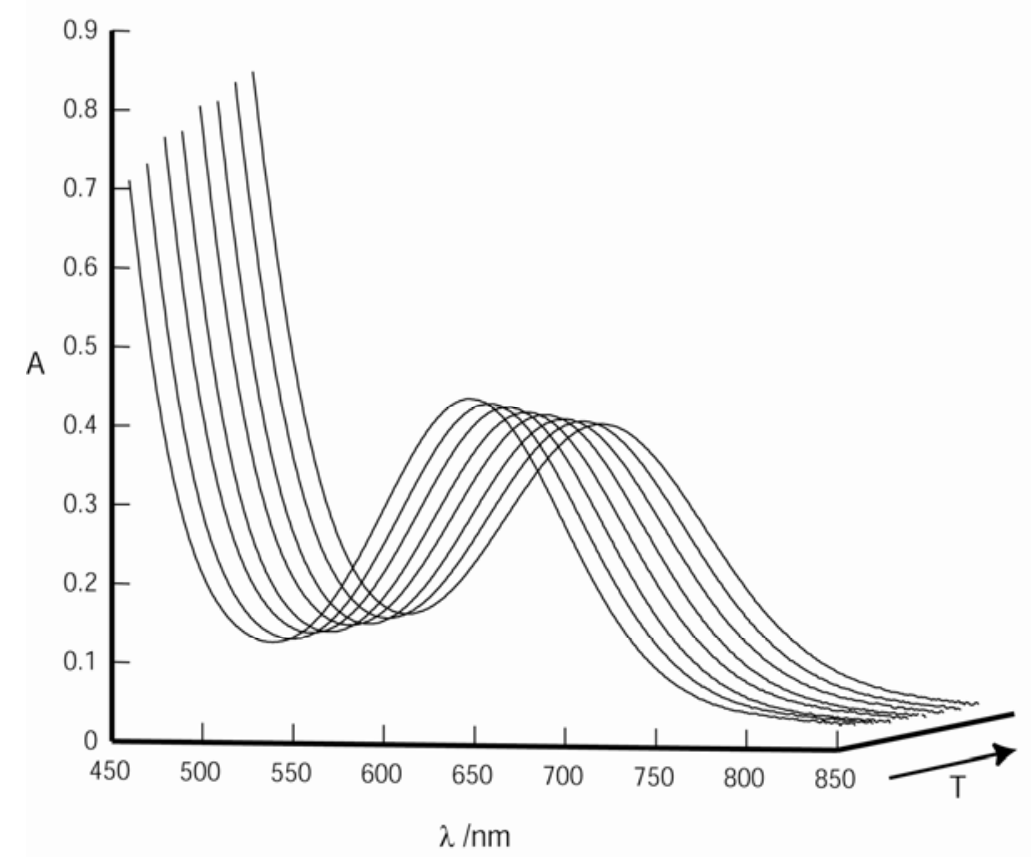

Figure S14. UV-visible spectra of $\mathbf{1}$ in $\left[\mathrm{C}_{5} \mathrm{e}_{3} \mathrm{am}\right]\left[\mathrm{Tf}_{2} \mathrm{~N}\right]$ at variable temperature. $\mathrm{c}(\mathbf{1})=0.26 \mathrm{mM}, \mathrm{T}=$ 9.7, 19.4, 29.1, 38.8, 48.4, 58.0, 67.7 and $77.2^{\circ} \mathrm{C}$. 


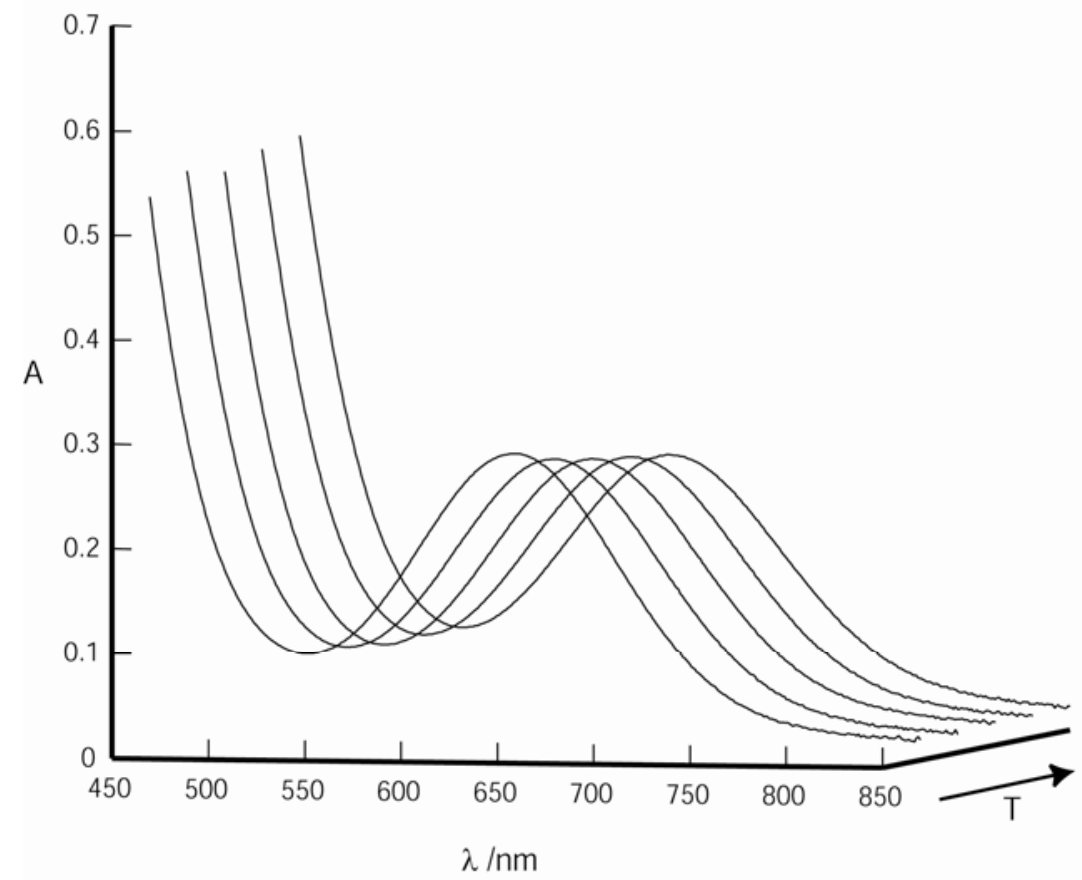

Figure S15. UV-visible spectra of $\mathbf{1}$ in $\left[\mathrm{C}_{5} \mathrm{e}_{3} \mathrm{am}\right]\left[\mathrm{Tf}_{2} \mathrm{~N}\right]$ at variable temperature. $\mathrm{c}(\mathbf{1})=0.18 \mathrm{mM}, \mathrm{T}=$ 29.0, 48.3, 58.0, 67.6 and $77.1{ }^{\circ} \mathrm{C}$.

Table S5 Absorbances at $638 \mathrm{~nm}$ of $\mathbf{1}$ dissolved in $\left[\mathrm{C}_{5} \mathrm{e}_{3} \mathrm{am}\right]\left[\mathrm{Tf}_{2} \mathrm{~N}\right]$.

\begin{tabular}{|rrrr|}
\hline $\mathrm{C}(\mathbf{1}) / \mathrm{mM}$ & $\mathrm{A}(638)$ & $1 / \mathrm{T} / \mathrm{K}^{-1}$ & $\mathrm{~T} /{ }^{\circ} \mathrm{C}$ \\
\hline 0.37 & 0.5942 & 0.003311 & 28.8 \\
0.37 & 0.5682 & 0.003112 & 48.1 \\
0.37 & 0.5435 & 0.002937 & 67.2 \\
0.37 & 0.5321 & 0.002857 & 76.8 \\
0.37 & 0.5552 & 0.003022 & 57.7 \\
0.37 & 0.5799 & 0.003207 & 38.6 \\
0.37 & 0.6082 & 0.003418 & 19.3 \\
0.37 & 0.6233 & 0.003533 & 9.8 \\
0.26 & 0.4145 & 0.003307 & 29.1 \\
0.26 & 0.4342 & 0.003534 & 9.7 \\
0.26 & 0.4229 & 0.003417 & 19.4 \\
0.26 & 0.4030 & 0.003205 & 38.8 \\
0.26 & 0.3847 & 0.003019 & 58.0 \\
0.26 & 0.3693 & 0.002853 & 77.2 \\
0.26 & 0.3774 & 0.002933 & 67.7 \\
0.26 & 0.3953 & 0.003109 & 48.4 \\
0.18 & 0.2885 & 0.003309 & 29.0 \\
0.18 & 0.2747 & 0.003110 & 48.3 \\
0.18 & 0.2679 & 0.003019 & 58.0 \\
0.18 & 0.2567 & 0.002854 & 77.1 \\
0.18 & 0.2626 & 0.002934 & 67.6 \\
\hline
\end{tabular}

